Volume 8, No.6, October - November 2020

International Journal of Bio-Medical Informatics and e-Health

Available Online at http://www.warse.org/IJBMIeH/static/pdf/file/ijbmieh01862020.pdf

https://doi.org/10.30534/ijbmieh/2020/01862020

\title{
Detection of a Human Body and object Sanitization using Deep Neural networks with Thermal Imaging
}

\author{
Aman Kumar, Sanjay Sharma \\ Student of Computer Science and Engineering, Shri Mata Vaishno Devi University, India, \\ kumaramanjha2901@ gmail.com. \\ Faculty of Computer Science and Engineering, Shri Mata Vaishno Devi University, India. \\ sanjay.sharma@smvdu.ac.in
}

\begin{abstract}
Thermal cameras or thermo graphic cameras are utilized in many different sectors like in industrial sector where it is used in metal reusing processing plants, water production line, detecting the heat of different machines and so on similarly, in healthcare it is used while taking out severe operations, detecting temperature of a human body and much more.it is also used by military for detecting intruders in nights and bad weather conditions where a normal camera cannot be used. Recent statistics indicate that the number of people diagnosed with COVID-19 is increasing exponentially, with more than 49 million confirmed cases; the disease is spreading to many countries across the world. In this paper, we proposed straightforward and quick recognition calculations into a cost effective detection of a body is properly sanitized or not sanitized using thermal imaging, this model can accomplish great precision in observing people warmth and distinguishing whether a body or an object is sanitized or not sanitized and can help to stop spreading of the deadly corona virus.
\end{abstract}

Key words: Corona virus (COVID-19), Deep learning, Thermal imaging system, Image Processing $\&$ Understanding.

\section{INTRODUCTION}

Corona virus disease (COVID-19) is an infectious disease caused by a newly discovered corona virus, which was first reported in Wuhan, Hubei province of China [1] [2] [3].it has spread all around the world at a high pace [4].United states of America has recently surpassed the number of 8.32 Million [5].The vast majority of the people who are affected with Covid-19 disease can get some respiratory sickness, fever, headaches, losing smelling senses and much more and it can be cured without any of the unique treatments. Some people with the previous respiratory problems or the older aged people can suffer more problems than an ordinary healthy human being, not proper care or medication can even led to deaths. So the most efficient approach is to tackle this easily spreadable virus by being well educated about this virus and what steps should be taken to prevent it [7]. Like being properly sanitized and being in less contact with the people as this virus is spread by the small droplets of saliva released from nose or mouth while coughing or sneezing. Therefore, it is especially important to wear a mask, maintain social distance and keep sanitizing yourself from time to time.

To date, there have been no detailed studies on the potential of artificial intelligence (AI) to detect COVID-19 automatically. So the best practice is to avoid it as much as possible and it can be done by getting in less contact with the people around, wearing masks and by process of sanitization.

The corona virus is very much easily transmissible and can stay up to days/hours in different objects like stainless steel, cardboard, clothes and many different products and can only be removed by proper sanitization, at recent times there are a lot of machines which helps for proper sanitization but nothing to detect whether a person or an object is fully sanitized or not. if we can detect whether an object is fully sanitized or not sanitized, then it can help to destroy the chain of transmission of this deadly virus. 
Deep learning, which is a well-known exploration region of man-made reasoning $(\mathrm{AI})$, helps in making different models for guaranteed output utilizing the different input data and attain high accuracy [8] [9]. Nowadays, different sectors are getting more dependent towards artificial intelligence and the healthcare sector is one of them. In healthcare, Deep learning is being used for numerous issues for example Pneumonia detection using chest X-rays [10], Brain disease detection using MRI scans [11], arrhythmia detection [12] [13], skin cancer detection and classification [14] [15] [16], Automated invasive ductal carcinoma detection [17] [18], automated segmentation of exudates [19], lung segmentation [20] [21] [22] and many more. The Covid-19 epidemic is increasing day by day and no vaccine till date has been identified and because of less number of testing kits and are not that much cost effective it is getting very difficult to identify and control this virus, to overcome this problem and give ideal help to the patients researchers in the field of artificial intelligence working for making easy and fast algorithm for the detection of covid-19.some of the works in this field are covid-19 detection using chest $\mathrm{x}$-rays [23], Thermal image for detection of body temperature and many more. Using artificial intelligence in the field of radiology can help to get more proper diagnosis [24].

In this study, easy and quick identification are implanted in a practical model for identification of sanitized images using thermal images. This model not only can be used on the humans but also on the objects to detect whether it is properly sanitized or not. It can be used to check whether the whole object is sanitized by detecting every point of the object on the same site, it can be used in a poor lightning condition and can achieve a high accuracy in detection of sanitized part. The study is divided in the accompanying manner. Section II gives the description of the component used for making the model. Section III, this section describes the algorithm used for detection of a human body or object Sanitized or not sanitized using Convolutional Neural networks with Thermal Imaging, Section IV reports some exploratory outcomes. At last in section $\mathrm{V}$, we reach a few conclusions and where can we use this model in the real world.

\section{THERMAL IMAGING FOR SANITIZATION DETECTION MODEL}

\subsection{Model Description:}

In this paper, we will take images from the thermal camera after the sanitization of the body and process that image with an CNN algorithm for the identification of human body and different objects whether they are properly sanitized or not and if it is partially sanitized then an alarm rings for the identification, as an average temperature of a human body varies from $97.7-99.5{ }^{\circ} \mathrm{F}$ and in thermal imaging it shows yellow-orange color and after the sanitization the image shows blue-purple color as shown in figure 1 , it shows a human arm before sanitization and after sanitization.

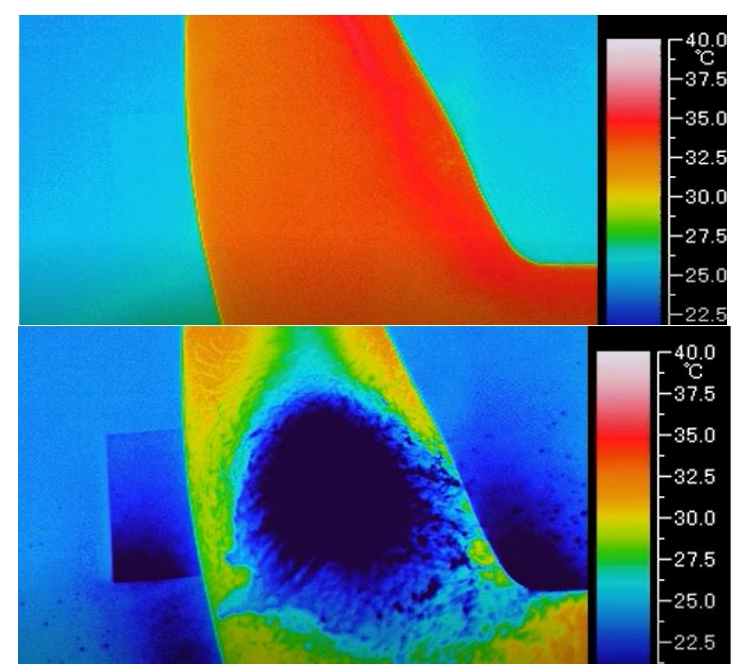

Figure 1: A human arm before sanitization and after sanitization

The model for the detection of a human body or an object sanitized or not sanitized is shown in figure 2 , framework is simple and straightforward, it requires a fine resolution thermal camera and a Laptop/PC where we can run deep neural networks for image classification.

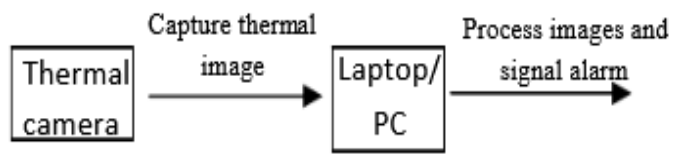

Figure 2: Thermal imaging for sanitization detection model 


\section{Thermal Camera}

A thermal camera, it is a device which creates an image using infrared radiations as the normal camera has a range of wavelength from 400-700 nanometers $(\mathrm{nm})$ whereas thermo graphic camera has a wavelength range of $1,000 \mathrm{~nm}$ to $14,000 \mathrm{~nm}$ These cameras are commonly used for detecting trespassers in environment [25],by military, detecting leakage in pipelines and much more because it can work in low lightening too. The infrared light was first founded by Sir William Herschel in 1960.Thermal imaging is tied in with changing that infrared light into electric signals and making an image utilizing that information. The basic standard today for thermal cameras is demonstrating hotter objects with a yellow-orange shade that gets more brilliant as the object gets more blazing. Colder objects are shown with a blue or purple color. Infrared energy has a frequency beginning at roughly 700 nanometers and reaches out to around $1 \mathrm{~mm}$. Wavelengths shorter than this start to be obvious by the unaided eye. Thermal imaging cameras utilize this infrared energy to make thermal images. The thermogram is then changed over to electrical signs to make a thermal image that we can see and interpret. The PC/laptop which is associated with the thermal camera at that point measures the pictures caught by thermal cameras.

\section{PC/Laptop}

Devices like pc or laptop will be used for the classification and processing of the input images and providing a suitable output. CNNs are utilized for image classification and recognition considering its high accuracy. CNN follows a stratified model that helps in building a network, like a channel, and finally releases a completely related layer where all the neurons are linked together, and the output is prepared. As per each person or object to be observed, it can effectively separate the images collected by the Thermal Camera, conveniently quantify caught thermal images with the algorithms. We can customize it with an alarm operator which will make some sound when the image detected is not- sanitized.

\section{ALGORITHM FOR DETECTION OF A HUMAN BODY AND OBJECT SANITIZATION USING DEEP NEURAL NETWORKS WITH THERMAL IMAGING}

In this study, basic, effective and fast algorithms are used for Detection of a human Body and object Sanitization using Deep Neural networks with Thermal Imaging has been proposed.

\subsection{Convolutional Neural Network used for Image Classification:}

Artificial Neural Networks are composed of artificial neurons that are motivated by our brain's organic neurons. Convolutional Neural Network $(\mathrm{CNN})$ is a modified feed-forward neural network variation that is widely used for tasks of image classification. Convolutional neural networks (ConvNets or CNNs) are one of the principle methods to do image Classification, object Detection, facial recognition and so on, are a portion of the regions where CNNs are broadly utilized.

\subsection{Working of a Convolution Neural Network:}

The accompanying tasks are the different $\mathrm{CNN}$ layers / steps:

$$
\begin{array}{ll}
\text { - } & \text { Convolution } \\
\text { - } & \text { Pooling } \\
\text { - } & \text { Flattening } \\
\text { - } & \text { Full Connection }
\end{array}
$$

We will now observe in depth what happens in each progression.

\subsubsection{Convolution}

Convolutional is the very first main layer to extricate highlights or attributes from an image input. By learning image features using small squares of input data, Convolutional preserves the relation between pixels. It is a numerical operation that requires two inputs, such as an image matrix and a filter or kernel. Figure 3 shows how the convolution functioning takes place. 


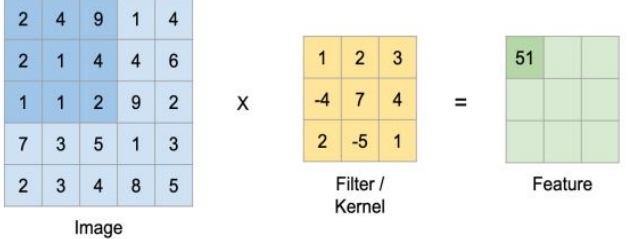

Figure 3: Convolutional functioning

Convolution losses data but reducing magnitude and being familiar with internal data is the argument here. Doing convolution can support image honing, edge discovery, blurring and other image processing tasks with different types of filters.

\subsubsection{Pooling}

The process of pooling helps to decrease the number of limits when the picture is large. Similarly, subsampling called Spatial Pooling shortens the dimensionality of each part map but contains crucial details. Pooling is essentially categorized into three types:

- $\quad$ Max Pooling (used mostly)

- Sum Pooling

- Average Pooling

Max pooling is an example-based measure for deconvolution. It is completed by applying a $\mathrm{N} \times \mathrm{N}$ max filter over the image, which selects the most noteworthy pixel value in each step and produces the map of the function. Essentially, the average and total of pixel values are taken into the function chart in the sum and average pooling.

Figure 4 Shows the process of Max Pooling:
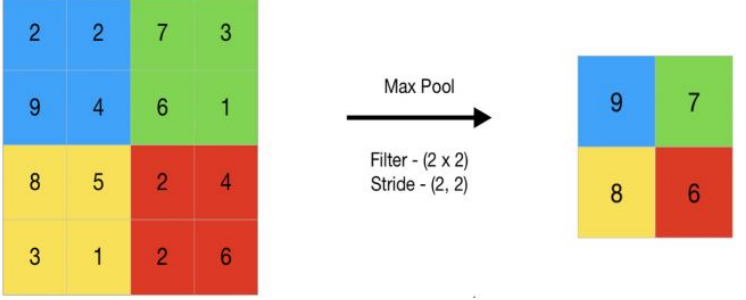

Figure 4: Max pooling

\subsubsection{Flattening}

We require a solitary column vector of the image pixels to implement our feature maps into artificial neural networks. We are flattening our function maps into column-like vectors, as the name suggests.
Figure 5 shows the flattening process.

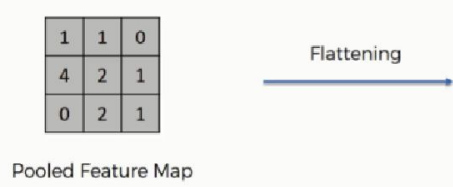

Figure 5: Flattening process

\subsubsection{Full Connection}

From the first convolution / pooling layer, the fully connected layer takes the contribution and gives a $\mathrm{N}$ dimensional vector where $\mathrm{N}$ is the number of groups to be graded. Subsequently, the layer calculates the highlights most aligned with a given class depending on the neuron probabilities.

Figure 6 In a neural network, the fully connected layer is shown.

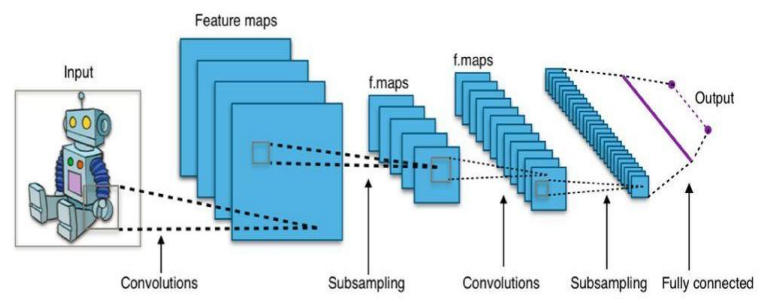

Figure 6: Fully Connected layers

\subsubsection{Flow chart for the model:}

Figure 7 shows the flow chart of the model.

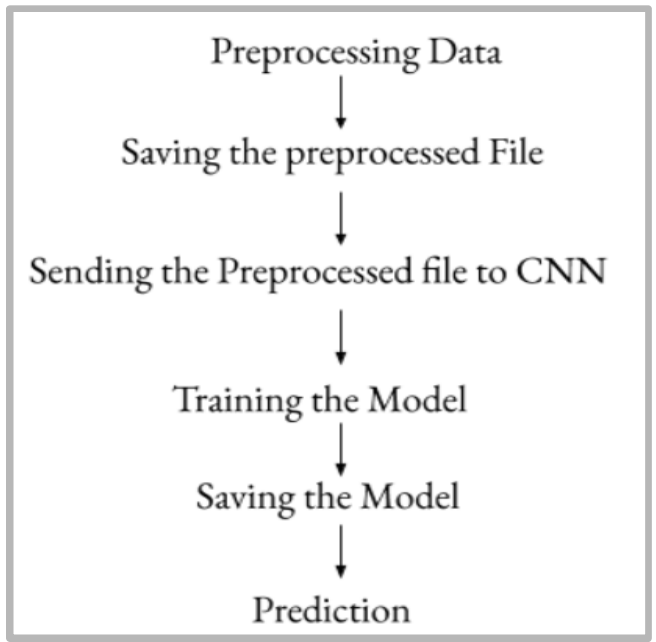

Figure 7: Flow chart of model 


\subsubsection{Steps to be followed for the system:}

We have divided the making of the model in different phases as given below to detect whether an object or a human body is sanitized using thermal images.

-Phase 1: Instating the input pictures and parameters which are required for the framework.

-Phase 2: Saving the processed data and separate them along their respective classes.

-Phase 3: Utilizing the convolutional neural network for discovering the predictions

-Phase 4: Train the model and acquire good accuracy. -Phase-5: Saving model for future predictions of testing images.

-Phase 6: Assess outcomes with the metrics like precision, accuracy, f1 score and recall.

\subsubsection{Phase wise explanation of algorithm of model}

The stepwise explanation of algorithm of model:

Phase 1: In computer vision, the biggest problem we face is the huge sizes of the input images.so to overcome this issue we follow the process of preprocessing as the data can be exceptionally large. Let's take an example, the picture size is 1000x1000x3 so the feature size will also be gigantic to send to a deep convolutional neural network. as the image is divided into three channels which is RGB where $R$ is red, $G$ is green and $B$ is blue. As taking in view of absence of the computational limits we then have to characterize a single channel while reading the image other than this there is one more issue of range of image. The informational index can contain pictures that are extraordinarily enormous in width and tallness. The width of the image can be 1000 and the stature of the image can be 700 which is amazingly generous to measure and needs significantly more computational ability to enlist a few pictures which is very tedious and a misuse of memory. So, we will have to resize the input images so that our machine can handle the input data with less memory requirement and less GPU. For handling these issues during processing of these pictures it would be rejected in such a manner that only a single colored channel remains.

Figure 8 shows thermal images of a person and a person with a dog.
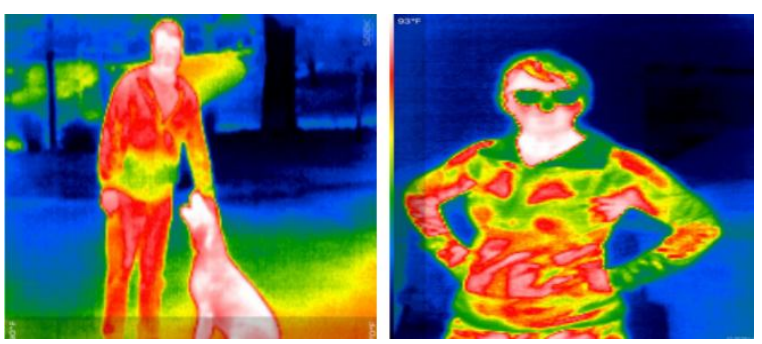

Figure 8: Thermal image of a person and a person with a dog.

Phase 2: In this phase we will save the Refined data and for single processed images it gets saved alongside their respective classes. By the given dataset only the sanitized and non - sanitized pictures are selected for future processing. We also need to neglect the pictures which do not have any of the class labels. Then the saved pictures are further sent to Deep Neural Networks.

Figure 9 shows image after sanitization.

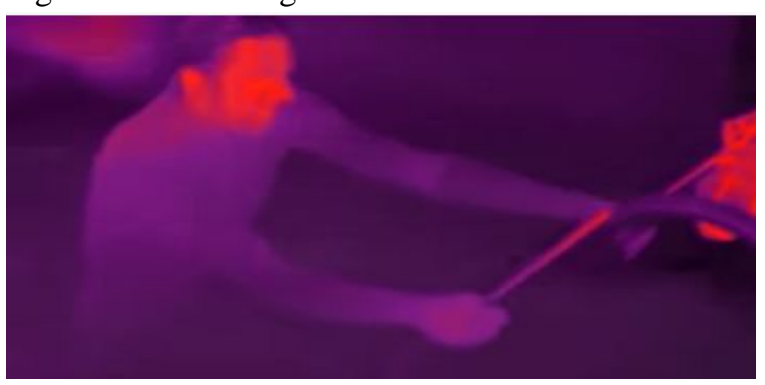

Figure 9: Image after sanitization.

Phase 3: In this phase, we will be sending the preprocessed data to a convolutional neural network. In a Computational neural network, there are 3 sorts of layers first is a convolutional layer, second is a pooling layer and third is a fully connected layer.

Firstly, the convolutional layer, using a proper example our model is explained here. Think that we are having a $6 \times 6$ grayscale image as figure 3 which is just a single channel. Then we have a $3 \times 3$ filter, which accumulate with $6 \times 6$ input image as shown in Figure 10 to give a $4 \times 4$ output as figure 6 as shown in figure11,where first element of the output layer is calculated as the sum of element wise product of $6 \times 6$ input image convolving with $3 \times 3$ filter taking Example, $3 \times 1+0 \times 0+1 \times-1+1 \times 1+5 \times 0+8 \times-$ $1+2 \times 1+7 \times 0+2 \times-1=-5$.. Similarly, Second and other elements are also extracted by same element wise product sum methodology by shifting the filter one unit right. 
In normal form, we can write $(\mathrm{a} x$ a) as input convolving with $\mathrm{a}(\mathrm{b} \mathrm{x}$ b) filter which will give output as:

$(a-b+1) \times(a-b+1)$

\begin{tabular}{|l|l|l|l|l|l|}
\hline 3 & 0 & 1 & 2 & 7 & 4 \\
\hline 1 & 5 & 8 & 9 & 3 & 1 \\
\hline 2 & 7 & 2 & 5 & 1 & 3 \\
\hline 0 & 1 & 3 & 1 & 7 & 8 \\
\hline 4 & 2 & 1 & 6 & 2 & 8 \\
\hline 2 & 4 & 5 & 2 & 3 & 9 \\
\hline
\end{tabular}

Figure 10: Convolving a $6 \times 6$ input image with a $3 \times 3$ filter

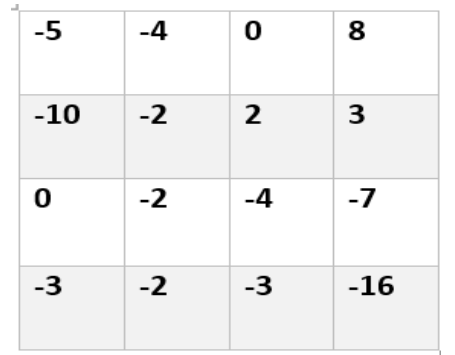

Figure 11: Output generated of $4 \times 4$ matrix after convolving $6 \times 6$ image with a $3 \times 3$ filter

One significant drawback during this process is the decrease in the size of the image. To overcome this problem we use the process of padding where an extra border is added. For example, when the input image is $6 \times 6$ matrix after applying padding it changes to a $8 \times 8$ matrix and after convolving it with a $4 \times 4$ filter we get back again an output of $6 \times 6$ matrix image so there will be no loss in the information of the input image.

In general form, we can write ' $\mathrm{x} x$ a' as input image and ' $\mathrm{b} \times \mathrm{b}$ ' as filter and after applying padding ( $\mathrm{p}$ ) and convolving input image with filter we get output as:

$(a+2 p-b+1) \times(a+2 p-b+1)$

To decrease the image size stride is a significant and helpful component in CNN. In general form when sride is denoted as ' $\mathrm{k}$ ' we get output as:

$[((a+2 p-b) / k)+1] \times[(a+2 p-b) / k)+1]$
Subsequently, the equation will look like 1 after the addition of bias in the equation. Then it will be passed through a ReLU function. Where 'bi' is bias term, 'xi' is input picture and ' $\mathrm{zi}$ ' is the filter:

$\mathrm{Zi}+=$ bi + xi x wi

$\operatorname{Relu}(z i)=\max (0$, zi $)$

The pooling layers are used for decreasing the picture size and for speeding up the computation considering a 4x4 matrix as shown in figure 12 .

Figure 13 shows max pooling.

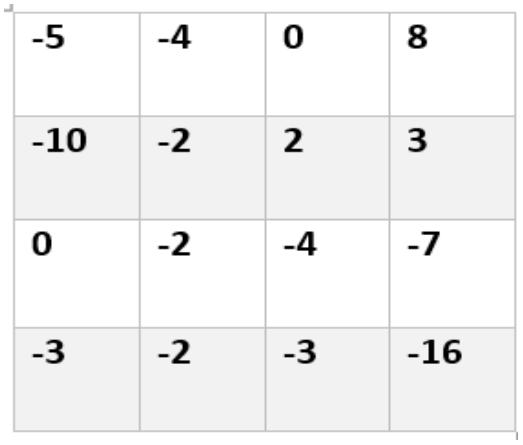

Figure 12: Image for pooling layers
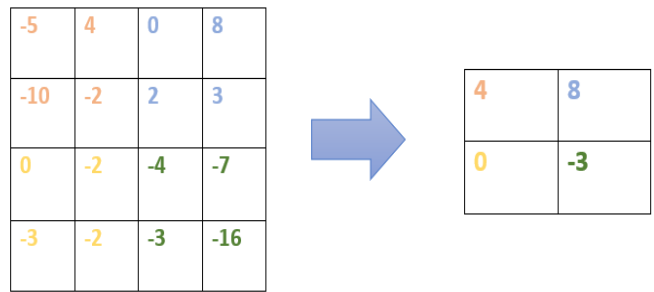

Figure 13: Max Pooling.

As in fig 13 for each successive $2 \times 2$ matrix the biggest value is extracted, and both the filter and stride are applied as size 2 units. Suppose a pooling layer is $\mathrm{xf} * \mathrm{xg} * \mathrm{xh}$ then we will set an output as $[\{(x f-b) / k+1) \times(x g-b) / k+1 \times x h]$. At that point, we will apply convolution $=\mathrm{s}$ and pooling again and again to extricate much more information. Then the extracted features or information are flattened to build a one single layer which will then feeded in a fully connected neural network. At that point after applying the softmax, the ideal outcome that is sanitized or not sanitized images is found. 
Phase 4: We need our model to be trained until the system loss is reduced to a particular level and we do not find any substantial amount of loss change.

Phase 5: After training the model we must save the model for the testing in future. Then the model is utilized to find out whether the given images are sanitized.

Phase 6: Now we will predict pictures utilizing the last layer. After the successful forecast for the testing pictures we will find out Precision, accuracy, recall and $\mathrm{f} 1$ score measures of the model.

\subsection{Evaluation Metrics}

It is to access the reliability of the proposed model. These matrices are calculated based on True Positive (TP), True negative (TN), False positive (FP) and False negative (FN) scores:

Accuracy: This metric measures the percentage of correctly identified cases relative to the entire dataset. The ML algorithm performs better if the accuracy is higher. Accuracy is a significant measure for a test dataset that includes a balanced class, it is calculated as shown in figure 13:

$$
\text { accuracy }=\frac{T P+T N}{T P+F N+T N+F P}
$$

Figure 13: Accuracy metric

Precision: It is a metric that assesses the amount of accurate positive predictions made, measured as the ratio of correct prediction of a body sanitized that were true positives divided by the total number of positive examples that were predicted, it is calculated as shown in figure 14 :

$$
\text { Precision }=\frac{T P}{T P+F P}
$$

Figure 14: Precision metric

Recall: It is a statistic that measures the amount of accurate positive forecasts made from all positive predictions that may have been made. It is measured as the ratio of positives correctly recognized as true positives, divided by the sum of actual positives and false negatives, it is calculated as shown in figure 15:

$$
\text { Recall }=\frac{T P}{T P+F N}
$$

\section{Figure 15: Recall metric}

F-measure: This is a combination of precision and recall that provides a significant measure for a test dataset that includes an imbalance class, it is calculated as shown in figure 16 :

$$
F 1=2 \cdot \frac{\text { precision } \cdot \text { recall }}{\text { precision }+ \text { recall }}
$$

Figure 16: F-measure metric

\section{RESULTS AND DISCUSSION}

In this Segment, we briefly depict the proposed Detection of a Human Body and object Sanitization using Deep Neural networks with Thermal Imaging model for the human sanitization detection part we take the thermal image of the person just after the sanitization if the person is properly sanitized then he/she is allowed to move forward but if the person is not properly sanitized or partially sanitized an alarm sound can be generated. For the object part same as above we will take the thermal images just after the sanitization process and check in the neural network if the object is sanitized or not sanitized. For implementing this model in factories or some other places we can just take a lot of images, divide it into sanitized and not sanitized objects and then train the model and get a high accuracy. As a lack of enough dataset can lead to the model overfitting so to come up with that we can use data augmentation where we can increase the number of training dataset images and then train the model to get an high and good accuracy [26].

\section{Some places where we can use this model are}

As we know there are lot of places where there is a lot of people come and go and a human eye cannot check whether a person is fully sanitized or not as the 
person can be a corona carrier so this model can be used there to check whether the person is sanitized and if not then an alarm can be placed or a person can check on the pc/laptop to identify the person.

Same as above in many factories where the product is handmade their sanitization of the product is very much necessary so getting thermal images just after sanitization can check whether the product is fully sanitized or not sanitized.

\section{CONCLUSION}

According to this study, a Convolutional Neural Networks based technique has been used for detection of a human body or object sanitized or not sanitized. A system is developed to help the people to get aware that they are sanitized and help to break a coronavirus chain. The best practice and currently the only way to get rid of covid-19 is to not let it spread and it can only be done by taking high precautions. By taking some random images anyone can detect whether an object or a human body is sanitized or not sanitized. The development of algorithms in this field can be highly beneficial for providing good healthcare services. As so far not much work has been done in this domain. Our proposed algorithm can be effective to detect whether a human body or an object is sanitized.

\section{REFERENCES}

[1] Wu, F., Zhao, S., Yu, B., et al. (2020). A new coronavirus associated with human respiratory disease in China, Nature, 579(7798), 265-269.

[2] Huang, C., Wang, Y., et al. (2020). Clinical features of the patients who got infected with novel coronavirus in Wuhan, China. The Lancet, 395(10223), 497-506.

[3] World Health Organization. (2020). Disease outbreak news, World Health Organization (WHO).

[4] Wu, Z., McGoogan, J. M. (2020). Characteristics and important lessons to learn from coronavirus disease 2019 (COVID-19) outbreak in China.

[5] Holshue, M. L., DeBolt, C., et al. (2020). First case of 2019 novel coronavirus in the United States, New England Journal of Medicine.
[6] Kong, W., \& Agarwal, P. P. (2020). Chest images of COVID-19 infection, Cardiothoracic Imaging, 2(1), e200028.

[7] Singhal, T. (2020). A Review of Coronavirus Disease-2019 (COVID-19), The Indian Journal of Pediatrics, 1-6.

[8] LeCun, Y., Bengio, Y., \& Hinton, G. (2015). Deep learning nature, 521(7553), 436-444.

[9] Krizhevsky, A., Sutskever, I., \& Hinton, G. E. (2012). ImageNet classification with deep convolutional neural networks, this report was a breakthrough that used convolutional nets to almost halve the error rate for object recognition (pp. 10971105).

[10] Rajpurkar, P., Irvin, J., et al. (2017). Chexnet: Radiologist-level pneumonia detection on chest $x$ rays with deep learning, arXiv preprint arXiv:1711.05225.

[11] Talo, M., Yildirim, O., Baloglu, U. B., Aydin, G., \& Acharya, U. R. (2019). Multiclass brain disease detection using MRI images with the use of computational neural networks, Computerized Medical Imaging and Graphics, 78, 101673.

[12] Hannun, A. Y., Rajpurkar, P., Haghpanahi, M., Tison, G. H., Bourn, C., Turakhia, M. P., \& Ng, A. Y. (2019). Cardiologist-level arrhythmia detection and classification in ambulatory electrocardiograms using a deep computational neural network, Nature medicine, 25(1), 65.

[13] Yıldırım, Ö., Pławiak, P., Tan, R. S., \& Acharya, U. R. (2018). Arrhythmia detection using deep convolutional neural networks with ECG signals, Computers in biology and medicine, 102, 411-420.

[14] Esteva A, Kuprel B, Novoa RA, et al. Dermatologist-level classification of skin cancer with deep neural networks, Nature. 2017;542(7639):115-118.

[15] Mahamadul Hasan, Surajit Das Barman, Samia Islam, Ahmed Wasif Reza. Skin cancer detection using convolutional neural network, International Conference on computing and artificial intelligence ICCAI 2019

[16] Acharya, U. R., Oh, S. L., Hagiwara, Y., Tan, J. H., Adam, M., Gertych, A., \& San Tan, R. (2017). A deep convolutional neural network model to classify heartbeats, Computers in biology and medicine, 89, 389-396.

[17] Celik, Y., Talo, M., Yildirim, O., Karabatak, M., \& Acharya, U. R. (2020). Automated Invasive 
Ductal Carcinoma Detection Based Using Deep Transfer Learning with Whole-Slide Images, Pattern Recognition Letters.

[18] Cruz-Roa, A., Basavanhally, A., et al. (2014, March), Automatic detection of invasive ductal carcinoma in whole slide images with convolutional neural networks, In Medical Imaging 2014: Digital Pathology (Vol. 9041, p. 904103). International Society for Optics and Photonics.

[19] Tan, J. H., Fujita, H., Sivaprasad, S., Bhandary, S. V., Rao, A. K., Chua, K. C., \& U. Rajendra Acharya (2017). Automated segmentation of exudates, haemorrhages, microaneurysms using single convolutional neural networks, Information sciences, 420, 66-76.

[20] Gaál, G., Maga, B., \& Lukács, A. (2020). Attention U-Net Based Adversarial Architectures for Chest X-ray Lung Segmentation, arXiv preprint arXiv:2003.10304,23 Mar 2020.

[21] Souza, J. C., Diniz, J. O. B., Ferreira, J. L., da Silva, G. L. F., Silva, A. C., \& de Paiva, A. C. (2019).Method for lung segmentation and reconstruction in chest $\mathrm{X}$-ray using deep computational neural networks, Computer methods and programs in biomedicine, 177, 285-296.

[22] N. C.F.Codella, Q. B. Nguyen, S. Pankanti, D. Gutman, B. Helba, A. Halpern, J. R. Smith (2017).

Deep learning ensembles for melanoma recognition in dermoscopy images, IBM Journal of Research and Development, 61(4/5), 5-1.

[23] Tulin Ozturk,Muhammed Talo,Azra Yildirim,Ulas Baran Baloglu (2020) . Automated Detection of COVID-19 Cases Using Deep Neural Networks with $\mathbf{X}$-ray Images, Computers in Biology and Medicine.

[24] Caobelli, F. (2020). Artificial intelligence in medical imaging: Game over for radiologists? European journal of radiology, 126, 108940.

[25] Wai Kit Wong, Poi Ngee Tan, Chu Kiong Loo and Way Soong Lim 2009, An Effective Surveillance System Using Thermal Camera, International Conference on Signal Acquisition and Processing, ICSAP 2009

[26] Agnieszka Mikołajczyk, Michał Grochowski (2018) Data augmentation for improving deep learning in image classification problem. 